\title{
COVID-19 Vaccine in Veterans with Multiple Sclerosis: Protect the Vulnerable
}

\author{
Francesca Bagnato, MD, PhD; and Mitchell Wallin, MD, MPH
}

Older veterans with progressive MS and associated comorbidities are at higher risk of death should they be infected by COVID-19 and we urge health care providers to educate every veteran about the benefits of being vaccinated against COVID-19.

Francesca Bagnato is the Associate Director of Research of the Multiple Sclerosis Center of Excellence East (MSCoE-East); a

Neurologist at Nashville Veterans Affairs

Medical Center (VAMC), and an Assistant

Professor at Vanderbilt University Medical Center in Tennessee. Mitchell Wallin is the Director of the MSCoE-East; a Neurologist at the Washington VAMC, and an Associate Professor at George Washington University in Washington, DC. Correspondence: Francesca Bagnato (francesca.bagnato@ va.gov)

Fed Pract. 2021;38(suppl 1). Updated online April 21. doi:10.12788/fp.0113
This article has been updated to reflect new US Food and Drug Administration and Centers for Disease Control and Prevention recommendations to pause administration of the Johnson and Johnson Jansen (JNJ-78436735) COVID-19 vaccine. ${ }^{1}$

$\longrightarrow$ ince the outbreak of the pandemic caused by the severe acute respiratory syndrome coronavirus-2 (SARS-CoV-2), a plethora of studies have been performed to increase our knowledge of its associated illness COVID-19. ${ }^{2}$ There is no cure for COVID-19, which can be lethal. In the absence of a cure, preventive measures are of vital importance. In order to help prevent the spread of the virus, the Centers for Diseases Control and Prevention (CDC) advocates for: (1) the use of a face mask over the mouth and nose; (2) a minimum of 6-foot distance between individuals; and (3) avoidance of gatherings. As of March 2021, the US Food and Drug Administration (FDA) approved 3 vaccines for the prevention of COVID-19, under an emergency use authorization (EUA). ${ }^{3-5}$ As of April 13, 2021, the FDA has recommended pausing the administration of one of these 3 vaccines due to unexpected rare but potentially lethal adverse effects, as detailed later. ${ }^{1}$

\section{COVID-19 AND MULTIPLE SCLEROSIS}

Since the beginning of the pandemic, neurologists have faced a new challenge-determining whether persons with multiple sclerosis (pwMS) were more at risk than others of becoming ill from COVID-19 or were destined for a worse outcome. The National MS Society has advised a personalized approach in relation to particularly vulnerable persons when needed and has also initiated worldwide registries to collect information regarding incidence and outcome of COVID-19 in pwMS. Accordingly, through the MS Center of Excellence (MSCoE), the Veterans Health Administration (VHA) has established a national registry assembling data regarding COVID-19 in veterans with MS.
A recent descriptive literature review summarized the outcomes of 873 persons with both MS and COVID-19 and reported that about 36\% of COVID-19 cases were treated with B-cell depleting therapies (ocrelizumab or rituximab). ${ }^{6}$ This proportion was relatively higher when compared with other disease modifying agents. Of those who became infected with SARS-CoV-2, death from COVID-19 occurred in about 4\%, and an additional 3\% required assisted invasive or noninvasive ventilation. Persons reported to have passed away from COVID-19 generally were older; had progressive MS; or had associated comorbidities such as obesity, hypertension, heart or lung conditions, or cancers. Of these, $50 \%$ were not on any disease modifying agent, $25 \%$ were on B-cell depleting therapies (ocrelizumab or rituximab), and the remaining $25 \%$ were on various medications for MS. It is important to highlight that no formal statistical analyses were performed in this review. On the contrary, in the recently published Italian report on 844 pwMS who had suspected or confirmed COVID-19, the authors used univariate and multivariate models to analyze their findings and noted that the use of ocrelizumab was significantly associated with a worse clinical outcome. $^{7}$ These authors also identified age, sex, disability score, and recent (within 1 month) use of steroids as risk factors for a severe COVID-19 outcome. The incidence of death from COVID19 in this cohort was $1.54 \%$.

The recently published data from the North American Registry of the National MS Society based on 1,626 patients reported a 3.3\% incidence of death from COVID-19.8 The following factors were identified as risks for worse outcome: male sex, nonambulatory status, age, Black race, and cardiovascular disease. The use of rituximab, ocrelizumab, and steroids (the latter medication over the preceding 2 months) increased the risks of hospitalization for COVID-19. 


\section{COVID-19 VACCINES}

Of the 3 available vaccines, the PfizerBioNTech COVID-19 (BNT162b2) vaccine is approved for individuals aged $\geq 16$ years, while the Moderna COVID-19 (mRNA-1273) and the Johnson and Johnson (J\&J) Janssen (JNJ-78436735) COVID-19 vaccines are approved for individuals aged $\geq 18$ years, though the latter vaccine has been temporarily suspended..$^{1,3-5}$ The Emergency Use Authorizations were released following the disclosure of the results of 3 phase 3 clinical trials and several phase 1 and 2 clinical trials. . $^{9-16}$

The BNT162b2 vaccine from Pfizer-BioNTech encodes the SARS-CoV-2 full-length spike protein (S) in prefusion conformation locked by the mutation in 2 prolines. ${ }^{9}$ Differently from the BNT162b2 vaccine, the BNT162b1 vaccine encodes a secreted trimerized SARS-CoV-2 receptor-binding domain. The S-glycoprotein is required for viral entry, as implicated in host cell attachment, and is the target of the neutralizing antibodies. In a phase 1 clinical study on 195 volunteers treated with BNT162b1 (10 mg, $20 \mathrm{mg}$, $30 \mathrm{mg}$, or $100 \mathrm{mg}$ doses) or BNT162b2 (10 mg, $20 \mathrm{mg}$, or $30 \mathrm{mg}$ doses) vaccines or placebo 21 days apart, both the binding and neutralizing antibody response was found to be age and "somewhat" dose dependent. ${ }^{9}$

Higher neutralization titers were measured at day 28 and 35 (7 and 14 days after the second dose, respectively) and compared with titers of persons who recovered from a COVID-19 infection.8 Serum neutralization was measured using a fluorescence-based high-throughput neutralization assay, while binding activity was assessed using the receptor-binding domain (RBD)-binding or S1-binding IgG direct Luminex immunoassays.

The overall reactogenicity/immunogenicity profile of BNT162b2 administered twice (30 mg each time) led to its selection for the phase 3 clinical trial. ${ }^{9,10}$ In a large phase 3 clinical trial on 43,458 participants, the BNT162b2 vaccine given at $30 \mathrm{mg}$ doses 21 days apart conferred $95 \%$ clinical efficacy in reducing the likelihood of being affected by symptomatic COVID-19. ${ }^{10}$ No safety concerns to stop the trial were identified, though related severe and lifethreatening events were reported in $0.3 \%$ and $0.1 \%$ of the volunteers, respectively. We note that these incidence rates were the same for the treated and the placebo group.

The mRNA-1273 vaccine from Moderna also encodes the SARS-CoV-2 S-glycoprotein.
In a dose escalation phase 1 trial of 45 participants aged between 18 and 55 years $(25 \mathrm{mg}$, $100 \mathrm{mg}$ or $250 \mathrm{mg}$, given at days 1 and 29) and 40 participants aged $\geq 57$ years $(25 \mathrm{mg}$ and $100 \mathrm{mg}$, given at days 1 and 29), a dosedependent effect was observed for both binding (receptor-binding domain and S-2p IgG on enzyme-linked immunosorbent assay [ELISA]) and neutralizing antibodies (SARS-CoV-2 nanoluciferase high-throughput neutralization assay, focus reduction neutralization test mNeonGreen and SARS-CoV-2 plaque-reduction neutralization testing assay) development. ${ }^{11,12}$ The geometric mean of both binding and neutralizing antibodies declined over time but persisted high as late as 119 days after the first burst of $100 \mathrm{mg}$ dose. ${ }^{13}$ The same dose of the vaccine also elicited a strong $T$ helper-1 response with little T helper-2 response across all ages. ${ }^{11}$ The strength of the memory cellular response remains to be defined and is the subject of ongoing investigations. In a large phase 3 clinical trial with 30,420 participants, the Moderna COVID-19 mRNA-1273 vaccine, given 28 days apart at the dose of $100 \mathrm{mg}$, met $94.1 \%$ clinical efficacy in reducing the likelihood of being affected by symptomatic COVID-19. ${ }^{14}$

Less than $0.1 \%$ of volunteers in both groups withdrew from the trial due to adverse effects (AEs); $0.5 \%$ in the placebo group and $0.3 \%$ in the treated group had AEs after the first dose, which precluded receiving the second dose. ${ }^{14}$

The Johnson and Johnson/Janssen JNJ78436735 vaccine is based upon a recombinant, replication-incompetent adenovirus serotype 26 (Ad26) vector, which encodes the full-length, stabilized S-glycoprotein of SARSCoV-2. The currently reported results of the phase 1 and 2 clinical study indicated that 805 volunteers (402 participants between ages 18 and 55 years and 403 individuals aged $\geq 65$ years) were randomized to receive a single or double dose of either $5 \times 1010$ viral particles per $0.5 \mathrm{~mL}$ (low dose) or $1 \times 1011$ viral particles per $0.5 \mathrm{~mL}$ (high dose), each compared with a placebo group. Incidence of seroconversion to binding antibodies against the fulllength stabilized S-glycoprotein, as measured by ELISA, showed $\geq 96 \%$ seroconversion by day 29 after the first dose. The incidence of seroconversion to neutralizing antibodies was $\geq 90 \%$ as early as early as 29 days after the first of either dose. In this study, neutralization activity was measured using the wild-type virus 
microneutralization assay based on the Victo$\mathrm{ria} / 1 / 2020 /$ SARS-CoV-2 strain. ${ }^{15}$ We note that the data related to this study have been partially reported and additional information will be available when each participant will have received the second dose.

In a large phase 3 clinical trial with 40,000 participants aged between 18 and 100 years, the Johnson and Johnson/Janssen JNJ78436735 vaccine, given as single dose of $5 \times 10^{10}$ viral particles per $0.5 \mathrm{~mL}$, met $65.5 \%$ clinical efficacy in the likelihood of being affected by symptomatic COVID-19 $\geq 28$ days postimmunization. ${ }^{16}$ In this study, the vaccine efficacy was found to have a geographic distribution with highest efficacy in the US (74.4\%), followed by Latin America (64.7\%) where Brazil showed a predominance of the P2 COVID-19 lineage (64.7\%), and Africa (52\%) where the B.1.351 lineage was most frequent $(94.5 \%)$. The vaccine also proved to be effective in reducing the likelihood of asymptomatic seroconversion, as measured by the level of a non-S protein, eg, $0.7 \%$ of positive cases in the vaccine group vs $2.8 \%$ in the placebo group. Immunological data indicated that the vaccine response was mainly driven by T-helper 1 lymphocytes. As of Apri 13, 2021 the FDA has recommend suspending the administration of the J\&J/Janssen vaccine due to the occurrence of severe blood clots reported in a 6 subjects out of $\sim 6.8$ millions administered doses. ${ }^{5}$

It is noteworthy to highlight that all vaccines reduced the likelihood of hospitalizations and deaths due to COVID-19.

As of April 17, 2021, the CDC reports that more than 130 million (40\%) Americans, nearly $1 / 3$ of the population, have received at least 1 dose of any of the 3 available vaccines, including 4.6 million at the VHA. ${ }^{17}$ Using the Vaccine Adverse Event Reporting System and v-safe, the US is conducting what has been defined the most "intense and comprehensive safety monitoring in the US history." 18 Thus far, data affirm the overall safety of the available vaccines against COVID-19. Individuals should not receive the COVID-19 vaccines if they have had a severe allergic reaction to any ingredient in the vaccine or a severe allergic reaction to a prior dose of the vaccine. Additionally, individuals who have received convalescent plasma should wait 90 days before getting the COVID-19 vaccine.
VACCINATION FOR PERSONS WITH MS

PwMS or those on immunosuppressive medications were excluded from the clinical trial led by Pfizer-BioNTech. There is no mention of MS as comorbidity in the study from Moderna, although this condition is not listed as an exclusion criterion either. The results of the phase 3 clinical trial for the Johnson and Johnson vaccine are not fully public yet, thus this information is not known as well. As a result, the use of this vaccine in pwMS under immunomodulatory agents is based on previous knowledge of other vaccines. Evidence is growing for the safety of the BNT162b2 COVID-19 vaccination in pwMS. Data regarding COVID-19 efficacy and safety are still largely based on previous knowledge $n$ other vaccines. $^{19}$

Immunization of pwMS is considered safe and should proceed with confidence in those persons who have no other contraindication to receive a vaccine. A fundamental problem for pwMS treated with immunomodulatory or immunosuppressive medications is whether the vaccine will remain safe or be able to solicit an adequate immune response. ${ }^{20,21}$ As of the time of publication 2021, there is consensus that mRNA based or inactivated vaccines are also considered safe in pwMS undergoing immunomodulatory or immunosuppressive treatments. ${ }^{18-20}$ We advise a one- on-one conversation between each veteran with MS and their primary neurologist to understand the importance of the vaccination, the minimal risks associated with it and if any specific treatment modification should be made.

To provide guidance, the National MS Society released a position statement that is regularly updated. ${ }^{22}$ Given the risks associated with discontinuation of disease modifying agents, pwMS opting to receive a COVID-19 vaccine should continue taking their medications unless recommended otherwise by their primary neurologist. In addition, on the basis of available literature and the American Academy of Neurology recommendations on the use of vaccines in general, the following recommendations are proposed. ${ }^{20-23}$

Recommendation 1: injections, orals, and natalizumab. Given the risks associated with discontinuation of disease modifying agents, pwMS opting to receive a COVID-19 vaccine should continue taking their medications unless recommended otherwise by their primary neurologist. Neither delay in start nor adjustments in dosing 
or timing of administration are advised for pwMS taking currently available either generic or brand formulations of $\beta$ interferons, glatiramer acetate, teriflunomide, dimethyl or monomethyl fumarate, or natalizumab. ${ }^{22}$

Recommendation 2: anti-CD20 monoclonal infusions. As an attenuated humoral response is predicted in pwMS treated with anti-CD20 monoclonal infusions, coordinating the timing of vaccination with treatment schedule may maximize efficacy of the vaccine. Whenever possible, it is advised to be vaccinated $\geq 12$ weeks after the last infusion and to resume infusion 4 weeks after the last dose of the vaccine. PwMS starting anti-CD20 monoclonal infusions are advised to be fully vaccinated first and start these medications $\geq 2$ to 4 weeks later. ${ }^{22}$

Recommendation 3: alemtuzumab infusion. Given its effect on CD52+ cells, it is advised to be vaccinated $\geq 24$ weeks after the last infusion and to resume infusion 4 weeks after the last dose of the vaccine. PwMS starting alemtuzumab infusions are advised to get fully vaccinated first and start this medication 4 weeks or more after completing the vaccine. ${ }^{22}$

Recommendation 4: sphingosine 1 phosphate receptor modulators, oral cladribine, and ofatumumab. PwMS starting any of these medications are advised to be fully vaccinated first and start these medications 2 to 4 weeks after completing the vaccine. PwMS already on those medications are not advised to change the schedule of administration. When possible, though, one should resume the dose of cladribine or ofatumumab 2 to 4 weeks after the last dose of the vaccine.22

Notably, all these recommendations hold true when there is enough disease stability to allow delaying treatment. We also add that it remains unclear if persons with an overall very low number of lymphocytes will be able to elicit a strong reaction to the vaccine. Blood collection and analysis of white blood cell count and lymphocyte subset estimates should be obtained in those persons with a markedly suppressed immune system. Whenever possible, to maximize outcome, timing the vaccination with treatment should be considered in those persons with a markedly reduced number of T-helper 1 cells.

\section{Vaccination for Veterans}

Currently the VHA is offering to veterans the Pfizer and Moderna COVID-19 COVID-19 vaccines with FDA EUAs. In accordance with FDA regulations, the VHA has paused administration of the Johnson and Johnson/Janssen vaccine. The VHA has launched its vaccination program in December 2020 by first providing the vaccine to health care personnel, nursing home patients, spinal cord injury patients, chemotherapy patients, dialysis and transplant patients, as well as homeless veterans. Most VA health care systems have passed this phase and are now able to provide vaccines to veterans with MS.

In December 2020, the MSCoE released a position statement regarding the importance and safety of the COVID-19 vaccine for veterans with MS. ${ }^{24}$ This statement will be updated on a regular basis as new information becomes available from major organizations like the National MS Society, FDA, CDC, and World Health Organization $(\mathrm{WHO})$ or relevant literature.

\section{CONCLUSIONS}

Older veterans with progressive MS and associated comorbidities are at higher risk of death should they be infected by COVID-19. Fortunately, we live in a time where vaccines are recognized as a critical tool to prevent this infection and to significantly reduce its morbidity and mortality. Yet, hesitancy to vaccinate has been identified as one of the most important threats to public health by the WHO in $2019 .{ }^{25}$ Understandably such hesitancy is even more profound for the COVID-19 vaccine, which is being administered under an EUA. In light of this indecision, and given the current state of the pandemic, we urge health care providers to educate every veteran about the benefits of being vaccinated against COVID-19. Within the VHA, a solid campaign of vaccination has been put in place at an unprecedented speed.

Health care providers interacting with veterans with MS are encouraged to use the MSCoE website (www.va.gov/ms) for any questions or concerns, or to reach out to MSCoE staff. It is vitally important that our community of veterans receives appropriate education on the importance of this vaccination for their own safety, for that of their household and society.

\section{Author disclosures}

The authors report no actual or potential conflicts of interest with regard to this article.

\section{Disclaimer}

The opinions expressed herein are those of the authors and do not necessarily reflect those of Federal Practitioner, Frontline Medical Communications Inc., the US Government, or any of its agencies. This article may discuss unlabeled or investigational use of certain drugs. Please review the complete prescribing 
information for specific drugs or drug combinations-including indications, contraindications, warnings, and adverse effectsbefore administering pharmacologic therapy to patients.

\section{References}

1. Centers for Disease Control and Prevention. Recommendation to pause use of Johnson \& Johnson's Janssen COVID-19 vaccine. Updated April 16, 2021. Accessed April 20, 2021. https://www.cdc.gov/coronavirus /2019-ncov/vaccines/safety/JJUpdate.html

2. World Health Organization. Naming the coronavirus disease (COVID-19) and the virus that causes it. Accessed March 9, 2021. https://www.who.int/emergencies /diseases/novel-coronavirus-2019/technical-guidance /naming-the-coronavirus-disease-(covid-2019)-and-thevirus-that-causes-it

3. US Food and Drug Administration. Pfizer-BioNTech COVID-19 vaccine. Updated February 3, 2021. Accessed March 22, 2021. https://www.fda.gov/emergency - preparedness-and-response/coronavirus-disease -2019-covid-19/pfizer-biontech-covid-19-vaccine

4. US Food and Drug Administration. Moderna COVID-19 vaccine. Updated February 3, 2021. Accessed March 22, 2021. https://www.fda.gov/emergency-preparedness -and-response/coronavirus-disease-2019-covid-19 /moderna-covid-19-vaccine

5. US Food and Drug Administration. FDA issues emergency use authorization for third COVID-19 vaccine [press release]. Published February 27, 2021. Accessed March 22, 2021. https://www.fda.gov/news-events /press-announcements/fda-issues-emergency-use -authorization-third-covid-19-vaccine

6. Möhn N, Konen FF, Pul R, et al. Experience in multiple sclerosis patients with COVID-19 and disease -modifying therapies: a review of 873 published cases. J Clin Med. 2020;9(12):4067. Published 2020 Dec 16. doi:10.3390/jcm9124067

7. Sormani MP, De Rossi N, Schiavetti I, et al. Disease-modifying therapies and coronavirus disease 2019 severity in multiple sclerosis. Ann Neurol. 2021;89(4):780-789. doi:10.1002/ana.26028

8. Salter A, Fox RJ, Newsome SD, et al. Outcomes and risk factors associated with SARS-CoV-2 infection in a North American registry of patients with multiple sclerosis [published online ahead of print, $2021 \mathrm{Mar}$ 19]. JAMA Neurol. 2021;10.1001/jamaneurol.2021.0688. doi:10.1001/jamaneurol.2021.0688

9. Walsh EE, Frenck RW Jr, Falsey AR, et al. Safety and immunogenicity of two RNA-based Covid-19 vaccine candidates. N Engl J Med. 2020;383(25):2439-2450. doi:10.1056/NEJMoa2027906

10. Polack FP, Thomas SJ, Kitchin N, et al. Safety and efficacy of the BNT162b2 mRNA Covid-19 vaccine. N Engl J Med. 2020;383(27):2603-2615. doi:10.1056/NEJMoa2034577

11. Jackson LA, Anderson EJ, Rouphael NG, et al. An mRNA vaccine against SARS-CoV-2-preliminary Report. N Engl J Med. 2020;383(20):1920-1931. doi:10.1056/NEJMoa2022483

12. Anderson EJ, Rouphael NG, Widge AT, et al. Safety and immunogenicity of SARS-CoV-2 mRNA-1273 vaccine in older adults. N Engl J Med. 2020;383(25):2427-2438. doi:10.1056/NEJMoa2028436

13. Widge AT, Rouphael NG, Jackson LA, et al. Durability of responses after SARS-CoV-2 mRNA-1273 vaccination. N Engl J Med. 2021;384(1):80-82. doi:10.1056/NEJMc2032195

14. Baden LR, El Sahly HM, Essink B, et al. Efficacy and safety of the mRNA-1273 SARS-CoV-2 vaccine. N Engl J Med. 2021;384(5):403-416. doi:10.1056/NEJMoa2035389

15. Sadoff J, Le Gars M, Shukarev G, et al. Interim results of a phase 1-2a Trial of Ad26.COV2.S Covid-19 Vaccine [published online ahead of print, 2021 Jan 13]. N Engl J Med. 2021;NEJMoa2034201. doi:10.1056/NEJMoa2034201

16. Oliver SE, Gargano JW, Scobie H, et al. The Advisory Committee on Immunization Practices' interim recommendation for use of Janssen COVID-19 vaccine - United States, February 2021. MMWR Morb Mortal Wkly Rep. 2021;70(9):329-332. Published 2021 Mar 5. doi:10.15585/ mmwr.mm7009e4

17. US Centers for Disease Control and Prevention. COVID19 vaccinations in the United States. Updated March 21, 2021. Accessed March 22, 2021. https://covid.cdc.gov/ covid-data-tracker/\#vaccinations

18. Gee J, Marquez P, Su J, et al. First month of COVID19 vaccine safety monitoring - United States, December 14, 2020-January 13, 2021. MMWR Morb Mortal Wkly Rep. 2021;70(8):283-288. Published 2021 Feb 26. doi:10.15585/mmwr.mm7008e3

19. Achiron A, Dolev M, Menascu S, et al. COVID-19 vaccination in patients with multiple sclerosis: What we have learnt by February 2021 [published online ahead of print, 2021 Apr 15]. Mult Scler. 2021;13524585211003476. doi:10.1177/13524585211003476

20. Righi E, Gallo T, Azzini AM, et al. A review of vaccinations in adult patients with secondary immunodeficiency [published online ahead of print, $2021 \mathrm{Mar} 9]$. Infect Dis Ther. 2021;1-25. doi:10.1007/s40121-021-00404-y

21. Ciotti JR, Valtcheva MV, Cross AH. Effects of MS disease-modifying therapies on responses to vaccinations: A review. Mult Scler Relat Disord. 2020;45:102439. doi:10.1016/j.msard.2020.102439

22. National Multiple Sclerosis Society. COVID-19 vaccine guidance for people living with MS. Accessed March 22, 2021. https://www.nationalmssociety.org/coronavirus -covid-19-information/multiple-sclerosis-and-coronavirus /covid-19-vaccine-guidance

23. Farez MF, Correale J, Armstrong MJ, et al. Practice guideline update summary: vaccine-preventable infections and immunization in multiple sclerosis: report of the Guideline Development, Dissemination, and Implementation Subcommittee of the American Academy of Neurology. Neurology. 2019;93(13):584-594. doi:10.1212/WNL.0000000000008157

24. US Department of Veterans Affairs, Multiple Sclerosis Centers of Excellence. Coronavirus (COVID-19) and vaccine information. Updated February 25. 2021. Accessed March 9, 2021. https://www.va.gov/ms

25. World Health Organization. Ten threats to global health in 2019. Accessed March 18, 2021. https://www.who.int /news-room/spotlight/ten-threats-to-global-health-in-2019. 